\title{
Engineering statistical transmutation of identical quantum particles
}

\author{
Simone Barbarino, ${ }^{1,2}$ Rosario Fazio, ${ }^{3,4}$ Vlatko Vedral, ${ }^{5,6}$ and Yuval Gefen ${ }^{7}$ \\ ${ }^{1}$ SISSA, Via Bonomea 265, 34136 Trieste, Italy \\ ${ }^{2}$ Institute of Theoretical Physics, Technische Universität Dresden, 01062 Dresden, Germany \\ ${ }^{3}$ ICTP, Strada Costiera 11, 34151 Trieste, Italy \\ ${ }^{4}$ NEST, Scuola Normale Superiore \& Istituto Nanoscienze-CNR, 56126 Pisa, Italy \\ ${ }^{5}$ Centre for Quantum Technologies, National University of Singapore, 3 Science Drive 2, 117543 Singapore \\ ${ }^{6}$ Clarendon Laboratory, University of Oxford, Parks Road, OX13PU Oxford, United Kingdom \\ ${ }^{7}$ Department of Condensed Matter Physics, The Weizmann Institute of Science, 76100 Rehovot, Israel
}

(Received 28 June 2018; published 22 January 2019)

\begin{abstract}
A fundamental pillar of quantum mechanics concerns indistinguishable quantum particles. In three dimensions they may be classified into fermions or bosons, having, respectively, antisymmetric or symmetric wave functions under particle exchange. One of the numerous manifestations of this quantum statistics is the tendency of fermions (bosons) to antibunch (bunch). In a two-particle scattering experiment with two possible outgoing channels [C. K. Hong, Z. Y. Ou, and L. Mandel, Phys. Rev. Lett. 59, 2044 (1987)], the probability of the two particles to arrive each at a different terminal is enhanced (with respect to classical particles) for fermions, and reduced for bosons. Here we show that by entangling the particles with an external degree of freedom, we can engineer quantum statistical transmutation, which may cause fermions to bunch. Our analysis may have consequences on the observed fractional statistics of anyons, including non-Abelian statistics, with serious implications for quantum computing operations in the presence of external degrees of freedom.
\end{abstract}

DOI: 10.1103/PhysRevB.99.045430

\section{INTRODUCTION}

The wave function of two or more indistinguishable particles must be invariant, up to a phase, if two of them are exchanged [1,2]. This requirement, together with the spinstatistics theorem, leads in three dimensions to the identification of fermions and bosons, having antisymmetric or symmetric wave functions under particle exchange. Indistinguishability is a central pillar of quantum mechanics underlying most facets of many-particle physics. An elegant way to bring to light the correlations hidden in the symmetry of the wave function is through interferometry $[3,4]$. In a two-particle interferometry bosons bunch together, i.e., the probability of detecting two incoming particles in the same outgoing terminal is higher than its benchmark value for classical particles; by contrast, fermions tend to antibunch due to the Pauli principle: it is impossible to have coincident detections of two fermions with identical quantum numbers at the same terminal. The effect of quantum statistics in Hanbury Brown-Twiss interferometric setups [5-7] has been highlighted in several fermionic-optics experiments [8-13]. We also note that (anti)bunching can be modified by assigning an internal degree of freedom (e.g., spin) to the scattering particles $[14,15]$.

Is it possible to control and modify on-demand the quantum statistics of identical particles? Examples of emergent particles, whose quantum statistics differs from that of their constituents, range from Cooper pairs [16-18] to fractional statistics anyons. The latter could be viewed as fermions with attached flux lines, in the fractional quantum Hall regime $[19,20]$. In this work we show that it is possible to induce statistical transmutation in a controlled way, without resorting to a direct particle-particle interaction. Our analysis shows that it is possible to engineer statistical transmutation of two identical quantum particles by entangling them with an external quantum degree of freedom. We study a simple setup where quantum statistics plays a fundamental role: a twoparticle scattering experiment in the presence of an external degree of freedom, e.g., a qubit, to which the scatterer is coupled. The build up of entanglement in the course of the scattering process is the underlying mechanism, allowing one to engineer statistical transmutation.

In order to analyze these effects we resort to the simple arrangement depicted in Fig. 1(a), commonly known as a Hong-Ou-Mandel interferometer [21,22], which consists of two sources at west (W) and south (S) that emit each a single particle. Following the scattering event these two particles may arrive in correspondence of the detectors at north $(\mathrm{N})$ and/or east $(\mathrm{E})$. The probability $P^{(0)}(2,0)\left[P^{(0)}(0,2)\right]$ would be the probability of the two scattering particles arriving both at $\mathrm{N}(\mathrm{E})$. The probability $P^{(0)}(1,1)$, in contrast, would be the probability of the two scattering particles arriving each at different detectors. The single-particle scattering amplitudes denote the probabilities that a single particle emitted from terminal $i(i=\mathrm{W}, \mathrm{S})$ arrives in terminal $j(j=\mathrm{N}, \mathrm{E})$. For classical noninteracting particles, the two-particle probabilities [e.g., $\left.P^{(0, c l)}(2,0)\right]$ are calculated assuming two independent scattering processes. These classical probabilities are to be used as "benchmarks" for comparison with two-particle processes, where quantum statistics does play a role. For example, one finds that for fermions $P^{(0, c l)}(1,1)<P^{(0)}(1,1)$ while for bosons $P^{(0, c l)}(1,1)>P^{(0)}(1,1)$. Hereafter, such 

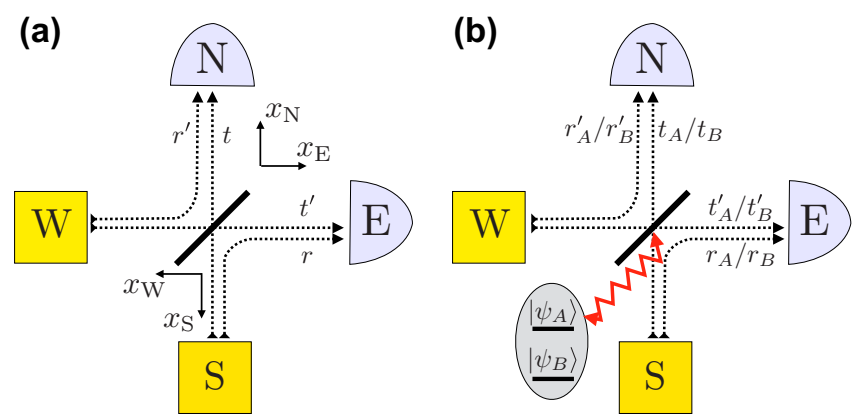

FIG. 1. (a) The standard Hong-Ou-Mandel interferometer: two particles emitted by the sources $\mathrm{W}$ and $\mathrm{S}$ go through the scattering region modeled by a beam-splitter, and they are collected by the two detectors $\mathrm{N}$ and $\mathrm{W}$. (b) The generalized Hong-Ou-Mandel interferometer: the beam splitter is coupled to an external qubit.

inequalities will be used to determine "femionic-like" or "bosonic-like" behavior. Statistical transmutation would mean that one type of behavior is transmuted to the other, i.e., anti-bunching is transmuted into bunching and vice versa.

Specifically, we consider a generalized Hong-Ou-Mandel interferometer [cf. Fig. 1(b)]. Here, the scatterer is coupled to a qubit. Consequently, additional quantum correlations between the incident particles and the qubit appear. Such correlations can lead to nonvanishing entanglement between the scattering particles and the qubit. One can then project the quantum state of the qubit onto a desired direction by performing a measurement of the qubit. This, in turn will generate correlations between the scattered particles, that may result in nontrivial effects such as statistical transmutation. This means that, following such a protocol, the probability of finding two fermions (bosons) in two different outgoing terminals, i.e., antibunching (bunching), may be suppressed (enhanced) in comparison with the classical benchmark, giving rise to statistical transmutation. Here, for the sake of clarity, we will mainly refer to fermionic particles.

Beyond our engineered statistical transmutation, we note here another intriguing effect: quantum correlations induced through the scatterer-qubit coupling may break the symmetry between the probability of collecting two particles in the $\mathrm{N}$ detector and collecting two particles in the $\mathrm{E}$ detector, $P(2,0) \neq P(0,2)$; cf. Fig. 1(b). This symmetry breaking is a manifestation of the entanglement between the scattered particles and the qubit.

\section{MODEL}

We consider a generalized Hong-Ou-Mandel interferometer; see Fig. 1(b). Particles are emitted from two sources, W (west) and S (south), and are measured, after passing through a scattering region, by two detectors placed at $\mathrm{N}$ (north) and $\mathrm{E}$ (east). Crucial for the present discussion are the properties of the scattering region. Differently from the standard setup of Fig. 1(a), the scattering of the incoming particles comprises a two-level system coupled to a beam splitter. The fermionic incoming particles are described by annihilation (creation) operators $a_{\mathrm{E}}\left(a_{\mathrm{E}}^{\dagger}\right)$ and $a_{\mathrm{S}}\left(a_{\mathrm{S}}^{\dagger}\right)$. The outgoing states are, in turn, described by annihilation (creation) operators $a_{\mathrm{N}}\left(a_{\mathrm{N}}^{\dagger}\right)$ and $a_{\mathrm{W}}$
$\left(a_{\mathrm{W}}^{\dagger}\right)$ and they satisfy the usual canonical anticommutation relations. The operators of the input states and output states are not independent but are related by a unitary transformation $s_{m}$ which is just the scattering matrix [23]

$$
\left(\begin{array}{c}
a_{\mathrm{E}} \\
a_{\mathrm{N}}
\end{array}\right)=\left(\begin{array}{cc}
r_{m} & t_{m}^{\prime} \\
t_{m} & r_{m}^{\prime}
\end{array}\right)\left(\begin{array}{c}
a_{\mathrm{S}} \\
a_{\mathrm{W}}
\end{array}\right),
$$

with $r_{m}=\sqrt{R_{m}} e^{i \theta_{m}}, t_{m}^{\prime}=\sqrt{T_{m}} e^{i \eta_{m}}, t_{m}=\sqrt{T_{m}} e^{i \eta_{m}}$, and $r_{m}^{\prime}=$ $-\sqrt{R_{m}} e^{i\left(2 \eta_{m}-\theta_{m}\right)} ; \eta_{m}, \theta_{m} \in[0,2 \pi)$. The scattering matrix carries an index $m$ associated with the presence of the two-level system. When the coupling between the qubit and the beam splitter is nonvanishing the scattering matrix will depend on the state of the qubit. Given a basis $\left|\psi_{m}\right\rangle$, with $m=A, B$, for the state of the qubit, we have $s_{A} \neq s_{B}$. All these parameters do depend on the state of the qubit.

Initially the particles and the qubit are in a factorized state

$$
\left|\Psi_{i}\right\rangle=\left|\psi_{0}\right\rangle|i\rangle
$$

where

$$
\left|\psi_{0}\right\rangle=\gamma_{A}\left|\psi_{A}\right\rangle+\gamma_{B}\left|\psi_{B}\right\rangle
$$

is the qubit quantum state $\left(\left|\gamma_{A}\right|^{2}+\left|\gamma_{B}\right|^{2}=1\right)$ and $|i\rangle$ is the particle state defined as

$$
|i\rangle=a_{\mathrm{W}}^{\dagger}\left(x_{\mathrm{W}}\right) a_{\mathrm{S}}^{\dagger}\left(x_{\mathrm{S}}\right)|0\rangle ;
$$

here the operator

$$
a_{\ell}^{\dagger}\left(x_{\ell}\right)=\int_{0}^{\infty} d k_{\ell} \epsilon_{\ell}\left(k_{\ell}\right) e^{-i k_{\ell} x_{\ell}} a_{\ell}^{\dagger}\left(k_{\ell}\right)
$$

with $\ell=\mathrm{W}, \mathrm{S}$, creates a fermionic particle in the source $\ell$ localized at $x_{\ell}$, which is the coordinate along the arm $\ell$, and it increases as we move along the arm toward the source. The operators $a_{\ell}\left(x_{\ell}\right)$ allow us to consider particles that arrive at the scatterer with a time delay which can be quantified in terms of the overlap integral

$$
|J|^{2}=\left|\int d k \epsilon_{\mathrm{W}}(k) \epsilon_{\mathrm{S}}^{*}(k) e^{i k\left(x_{\mathrm{W}}-x_{\mathrm{S}}\right)}\right|^{2} ;
$$

if $\epsilon_{\mathrm{W}}$ and $\epsilon_{\mathrm{S}}$ are two Gaussian wave packets of spatial width $\delta$ and central velocity $v$ such that $x_{\ell}=v \tau_{\ell}(\ell=\mathrm{W}, \mathrm{S})$, the overlap integral is [3]

$$
|J|^{2}=\exp \left[-\frac{v^{2}\left(\tau_{\mathrm{W}}-\tau_{\mathrm{S}}\right)^{2}}{2 \delta^{2}}\right] .
$$

For $|J|^{2}=1$ particles are indistinguishable, while for $|J|^{2}=$ 0 particles are classical. Following the scattering process, the final state of the system becomes

$$
\left|\Psi_{f}\right\rangle=\sum_{m=A}^{B} \gamma_{m} S_{m}|i\rangle\left|\psi_{m}\right\rangle,
$$

where $S_{m}=s_{m}^{(\mathrm{W})} \otimes s_{m}^{(\mathrm{S})}$ is the two-particle scattering matrix. We stress here that the final state $\left|\Psi_{f}\right\rangle$ is generically entangled. As we will see, this property is at the core of statistical transmutation. Assuming ideal detectors, we evaluate the probability that $n$ fermionic particles are revealed by the detector at $\mathrm{N}$ while $2-n$ particles are collected by the detector at W. Since the final state will be generically entangled, these 
probability will depend on the dynamics and measurements on the qubit. To this end, we will consider two different protocols for the dynamics of the qubit.

\section{A. Protocol 1}

Here, we evaluate the probability of particles to arrive at the detectors, independently of the state of the qubit. This can be done by tracing out the qubit degrees of freedom from the density matrix of the final state, $\rho_{f}=\left|\Psi_{f}\right\rangle\left\langle\Psi_{f}\right|$. This probability,

$$
P(n, 2-n)=\sum_{m=A}^{B}\left|\gamma_{m}\right|^{2} P_{m}(n, 2-n),
$$

can be expressed by the weighted average of the probabilities,

$$
P_{m}(n, 2-n)=\int d k d k^{\prime}\left|\left\langle n, 2-n ; k, k^{\prime}\left|S_{m}\right| i\right\rangle\right|^{2},
$$

where

$$
\begin{aligned}
& \left|2,0 ; k, k^{\prime}\right\rangle=1 / \sqrt{2} a_{\mathrm{N}}^{\dagger}(k) a_{\mathrm{N}}^{\dagger}\left(k^{\prime}\right)|0\rangle \\
& \left|0,2 ; k, k^{\prime}\right\rangle=1 / \sqrt{2} a_{\mathrm{E}}^{\dagger}(k) a_{\mathrm{E}}^{\dagger}\left(k^{\prime}\right)|0\rangle \\
& \left|1,1 ; k, k^{\prime}\right\rangle=a_{\mathrm{N}}^{\dagger}(k) a_{\mathrm{E}}^{\dagger}\left(k^{\prime}\right)|0\rangle
\end{aligned}
$$

A straightforward calculation yields

$$
\begin{aligned}
& P_{m}(1,1)=R_{m}^{2}+T_{m}^{2}+2 R_{m} T_{m}|J|^{2}, \\
& P_{m}(2,0)=P_{m}(0,2)=R_{m} T_{m}\left(1-|J|^{2}\right) .
\end{aligned}
$$

Equation (7) is readily understood. The trace over the qubit degrees of freedom suppresses all possible quantum correlations between the particles, and the final result is a weighted sum over the probabilities $P_{m}(n, 2-n)$ defined for a fixed qubit state. The limiting case of classical particles $P^{(c l)}(1,1)$ can be obtained, as expected, from $P_{m}(1,1)$ by setting $|J|^{2}=$ 0 , i.e., $\left.P_{m}^{(c l)}(1,1) \equiv P_{m}(1,1)\right|_{|J|^{2}=0}$, and employing Eq. (7). It is evident that protocol 1 cannot lead to any statistical transmutation. For fermionic particles we still have antibunching, i.e., the probability of collecting the two scattered particles into two different detectors is greater for fermions than for classical particles, $P(1,1)>P^{(c l)}(1,1)$.

\section{B. Protocol 2}

To demonstrate how quantum correlations between the qubit and the particles may induce bunching (even for fermions), we now resort to projecting the qubit onto a given state, $|\psi\rangle$, following the scattering process. We then evaluate the probability that $n$ particles are absorbed by the first detector while $2-n$ particles are collected by the other detector, obtaining

$$
P(n, 2-n ;|\psi\rangle)=\frac{\int d k d k^{\prime}\left|\sum_{m} \gamma_{m} \alpha_{m}^{(n)}\left\langle\psi \mid \psi_{m}\right\rangle\right|^{2}}{\sum_{\lambda=0}^{2} \int d k d k^{\prime}\left|\sum_{m} \gamma_{m} \alpha_{m}^{(\lambda)}\left\langle\psi \mid \psi_{m}\right\rangle\right|^{2}},
$$

with $\alpha_{m}^{(n)}=\left\langle n, 2-n ; k, k^{\prime}\left|S_{m}\right| i\right\rangle$. The denominator of Eq. (11) is a normalization factor which guarantees that $\sum_{n} P(n, 2-n ;|\psi\rangle)=1$; it is indeed equivalent to a post-selection procedure onto the final qubit state (cf. Appendix B). Protocol 2 does not have a classical counterpart.

Before proceeding, we observe that when the beam-splitter is not coupled to the qubit, i.e., $s_{A}=s_{B} \equiv s_{0}$, the final state $\left|\Psi_{f}\right\rangle$ is separable and there are no quantum correlations between particles and the qubit. In this case the two protocols are equivalent, i.e., $P(n, 2-n ;|\psi\rangle)=P(n, 2-n)$. For this reason, in the following, we assume that the beam splitter is nontrivially coupled to the qubit, i.e. $s_{A} \neq s_{B}$. As a concrete example, we consider here the special case where $R_{A}=$ $R_{B}=R$ and $T_{A}=T_{B}=T$. Under these assumptions, according to protocol- 1 we have $P_{A}(n, 2-n)=P_{B}(n, 2-n)=$ $P(n, 2-n)$ and, for classical particles, we have $P^{(c l)}(n, 2-$ $n)=\left.P(n, 2-n)\right|_{|J|^{2}=0}$. Furthermore the probabilities given in Eq. (11) can be cast in the form

$$
P(n, 2-n ;|\psi\rangle)=\mathcal{S}(n,|\psi\rangle) P(n, 2-n)
$$

with

$$
\begin{gathered}
\mathcal{S}(n,|\psi\rangle)=\frac{\left|\tilde{\gamma}_{A}\right|^{2}+\left|\tilde{\gamma}_{B}\right|^{2}+2\left|\tilde{\gamma}_{A}\right|\left|\tilde{\gamma}_{B}\right| \cos \varphi_{n, 2-n}}{\left|\tilde{\gamma}_{A}\right|^{2}+\left|\tilde{\gamma}_{B}\right|^{2}+2 \lambda\left|\tilde{\gamma}_{A}\right|\left|\tilde{\gamma}_{B}\right| \cos \varphi_{1,1}} ; \\
\lambda \equiv P(1,1)+2 P(2,0) \cos \left(\eta_{B}-\eta_{A}+\theta_{A}-\theta_{B}\right)
\end{gathered}
$$

with

$$
\begin{aligned}
& \varphi_{1,1}=\varphi_{0}+2\left(\eta_{A}-\eta_{B}\right) \\
& \varphi_{2,0}=\varphi_{0}+\eta_{A}+\theta_{A}-\left(\eta_{B}+\theta_{B}\right), \\
& \varphi_{0,2}=\varphi_{0}+3 \eta_{A}-\theta_{A}-\left(3 \eta_{B}-\theta_{B}\right)
\end{aligned}
$$

and $\tilde{\gamma}_{m}=\gamma_{m}\left\langle\psi \mid \psi_{m}\right\rangle \equiv\left|\tilde{\gamma}_{m}\right| e^{i \arg \left(\tilde{\gamma}_{m}\right)}, \varphi_{0}=\arg \left(\tilde{\gamma}_{A}\right)-\arg \left(\tilde{\gamma}_{B}\right)$. The coefficient $\mathcal{S}(n,|\psi\rangle)$, which depends on the number of particles detected, on the final state of the qubit, as well as on the phases of the scattering matrices and on the overlap integral between the incident particles, contains all the relevant information related to the modifications of the probability distribution generated by our protocol 2. It is straightforward to observe that protocol 2 can generate nontrivial effects when $\mathcal{S}(n,|\psi\rangle) \neq 1$. We note that $\mathcal{S}(n,|\psi\rangle) \neq 1$ as long as the final state of the system $\left|\Psi_{f}\right\rangle$ is entangled and the qubit state onto which we project is in a nontrivial superposition of the states $\left|\psi_{A}\right\rangle$ and $\left|\psi_{B}\right\rangle$. In order to quantify whether the final state $\left|\Psi_{f}\right\rangle$ is entangled or not, we construct the density matrix

$$
\rho_{f}=\left|\Psi_{f}\right\rangle\left\langle\Psi_{f}\left|=\sum_{m, m^{\prime}} \gamma_{m} \gamma_{m^{\prime}}^{*} S_{m}\right| i\right\rangle\left\langle i\left|S_{m^{\prime}}^{\dagger}\right| \psi_{m}\right\rangle\left\langle\psi_{m^{\prime}}\right| .
$$

The reduced density matrix of the qubit is then obtained by tracing out the particles from the whole density matrix $\rho_{f}$ :

$$
\rho_{f}^{r e d}=\left(\begin{array}{cc}
\left|\gamma_{A}\right|^{2} & \gamma_{A} \gamma_{B}^{*} \epsilon^{*} \\
\gamma_{A}^{*} \gamma_{B} \epsilon & \left|\gamma_{B}\right|^{2}
\end{array}\right)
$$

here $\left\langle i\left|S_{A}^{\dagger} S_{B}\right| i\right\rangle \equiv \epsilon$. A straightforward calculation shows that the final state $\left|\Psi_{f}\right\rangle$ is entangled if and only if $|J|^{2}<1$ and $\eta_{B}-\eta_{A}+\theta_{A}-\theta_{B} \neq 0$, provided that $\gamma_{A}, \gamma_{B} \neq 0$.

\section{RESULTS}

\section{A. Statistical transmutation}

We stress here again that statistical transmutation happens when the probability of finding two outgoing fermions in two 
different terminals exhibits bunching rather than the usual antibunching, i.e., $P(1,1 ;|\psi\rangle)$ is smaller than $P^{(c l)}(1,1)$. Indeed, protocol 2 allows one to transmute the statistics of the incoming particles in a controlled way by properly selecting the final state $|\psi\rangle$ onto which the qubit is projected. Importantly we observe that a necessary but insufficient requirement to obtain statistical transmutation is the presence of qubitparticles entanglement in the final state, i.e., $\mathcal{S}(n,|\psi\rangle) \neq 1$ (following the scattering).

This reinforces the intuition that, in order to have transmuted statistics, one should consider composite particles (formed by the fermions and the qubit). Note, however, that statistical transmutation becomes possible only when $\mathcal{S}(1,|\psi\rangle)<P^{(c l)}(1,1) / P(1,1)$. This last inequality leads to

$$
\frac{|J|^{2}}{2 P^{(c l)}(1,1)\left(1-|J|^{2}\right)}<\eta,
$$

where

$$
\eta=\frac{\left|\tilde{\gamma}_{A}\right|\left|\tilde{\gamma}_{B}\right|\left[\cos \left(\eta_{B}-\eta_{A}+\theta_{A}-\theta_{B}\right)-1\right] \cos \varphi_{1,1}}{\left|\tilde{\gamma}_{A}\right|^{2}+\left|\tilde{\gamma}_{B}\right|^{2}+2\left|\tilde{\gamma}_{A}\right|\left|\tilde{\gamma}_{B}\right| \cos \varphi_{1,1}} .
$$

In order to better understand the idea of statistical transmutation, we consider some examples. In the following, we choose as initial state of the qubit $\left|\psi_{0}\right\rangle=1 / \sqrt{2}\left(\left|\psi_{A}\right\rangle+\left|\psi_{B}\right\rangle\right)$ and we set the scattering matrix amplitudes $R_{m}=T_{m}=0.5$ with $m=A, B$ [this choice guarantees that statistical effects are maximized, i.e. $P_{m}(1,1)-P_{m}^{(c l)}(1,1)=2 R_{m} T_{m}|J|^{2}$ is maximum]; furthermore, without loss of generality, we set $\eta_{A}=\theta_{A}=0$. In Fig. 2(a) we consider the probability $P(1,1 ;|\psi\rangle)$ as a function of the phases $\eta_{B}$ and $\theta_{B}$ for a fixed final qubit state $|\psi\rangle=\left|\psi_{0}\right\rangle$. We observe the existence of a nonvanishing region in the parameter space $\left(\eta_{B}, \theta_{B}\right)$, where $P(1,1 ;|\psi\rangle)$ is smaller then $P^{(c l)}(1,1)$, and a region where statistical transmutation does not happen. Moreover,
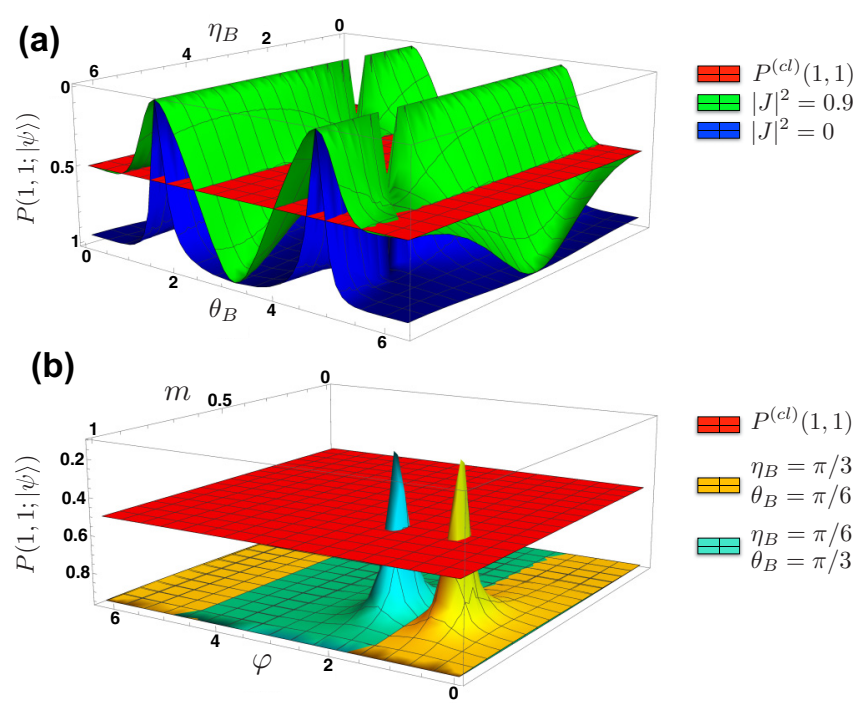

FIG. 2. Statistical transmutation, i.e., $P(1,1 ;|\psi\rangle)<P^{(c l)}(1,1)$ can be induced by selecting the final state $|\psi\rangle$ onto which the qubit is projected. (a) Statistical transmutation as a function of the scattering phases $\eta_{B}$ and $\theta_{B}$ with $\left|\psi_{f}\right\rangle=\left|\psi_{0}\right\rangle$. (b) Statistical transmutation as a function of the final state $|\psi\rangle=m\left|\psi_{A}\right\rangle+e^{i \varphi} \sqrt{1-m^{2}}\left|\psi_{B}\right\rangle$ for fixed values of the scattering phases.

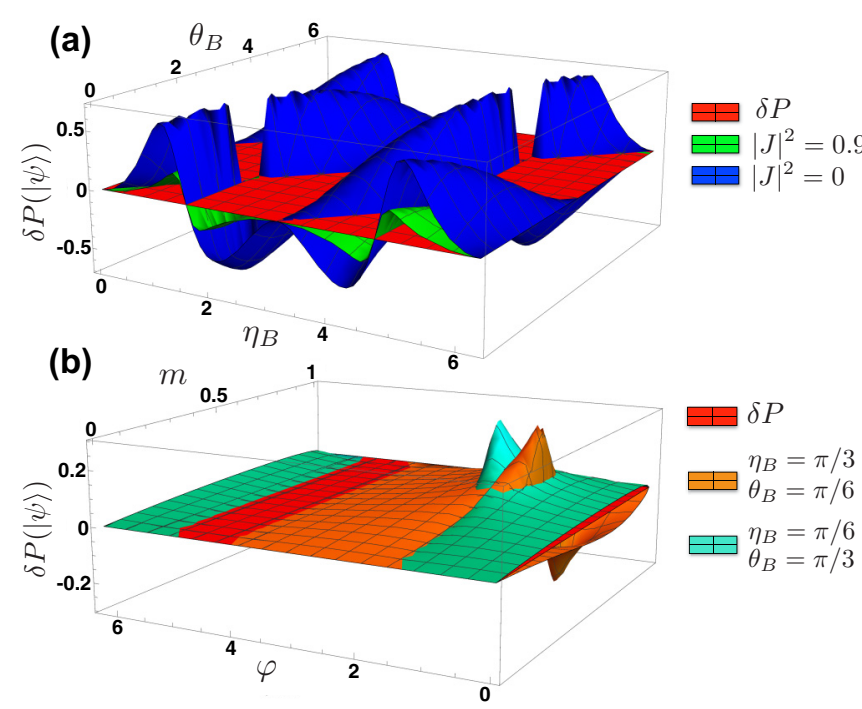

FIG. 3. The probabilities $P(2,0 ;|\psi\rangle)$ and $P(0,2 ;|\psi\rangle)$ of finding two outgoing fermions in the same terminal can be different. This effect can be quantified by $\delta P(|\psi\rangle)=P(2,0 ;|\psi\rangle)-P(0,2 ;|\psi\rangle)$. (a) $\delta P(|\psi\rangle)$ as a function of the scattering phases $\eta_{B}$ and $\theta_{B}$ with $\left|\psi_{f}\right\rangle=\left|\psi_{0}\right\rangle$. (b) $\delta P(|\psi\rangle)$ as a function of the final state $|\psi\rangle=$ $m\left|\psi_{A}\right\rangle+e^{i \varphi} \sqrt{1-m^{2}}\left|\psi_{B}\right\rangle$ for fixed values of the scattering phases.

from Fig. 2(a) the peculiar quantum nature of protocol 2 comes out. The probability $P(1,1 ;|\psi\rangle)$ with $|J|^{2}=0$, i.e., particles for which the Pauli principle does not apply, still exhibits enhancement or suppression with respect to $P^{(c l)}(1,1)$, signaling that the qubit gives rise to additional quantum correlations beyond the Pauli principle. Finally, in Fig. 2(b), we fix the scattering phases $\left(\eta_{B}, \theta_{B}\right)$, and we calculate the probability $P(1,1 ;|\psi\rangle)$ as a function of the final state $|\psi\rangle=$ $m\left|\psi_{A}\right\rangle+e^{i \varphi} \sqrt{1-m^{2}}\left|\psi_{B}\right\rangle$ onto which the qubit is projected. We then observe the existence of a nontrivial manifold of final states $|\psi\rangle$, which underlines the parameter space for statistical transmutation.

\section{B. Unitarity breaking}

Besides facilitating statistical transmutation, protocol 2 allows one to generate an asymmetry between the probabilities of collecting two particles in the $\mathrm{E}$ and in the $\mathrm{N}$ detector. Such an asymmetry is strictly forbidden for quantum particles, owing to the unitarity of the scattering matrix implying $P(2,0)=P(0,2)$. Below we discuss under which conditions $P(2,0 ;|\psi\rangle)$ and $P(0,2 ;|\psi\rangle)$ can be different. We argue that this effect corresponds to an effective breaking of the unitarity of the scattering matrix.

In order to study this effect quantitatively, we define the quantity $\delta P(|\psi\rangle)=P(2,0 ;|\psi\rangle)-P(0,2 ;|\psi\rangle)$, which can be expressed as

$$
\delta P(|\psi\rangle)=[\mathcal{S}(2,|\psi\rangle)-\mathcal{S}(0,|\psi\rangle)] P(2,0),
$$

and is nonvanishing when $\mathcal{S}(2,|\psi\rangle) \neq \mathcal{S}(0,|\psi\rangle)$. In view of Eq. (13), it is straightforward to see that $\delta P(|\psi\rangle)$ is nonvanishing as long as the final state $\left|\Psi_{f}\right\rangle$ is entangled.

In Fig. 3(a) we consider the asymmetry $\delta P(|\psi\rangle)$ as a function of the phases $\eta_{B}$ and $\theta_{B}$ for a fixed final qubit state $|\psi\rangle=\left|\psi_{0}\right\rangle$, and we observe the existence of a nonvanishing 
region in parameter space where $\delta P(|\psi\rangle)$ is positive, i.e., $P(2,0 ;|\psi\rangle)>P(0,2 ;|\psi\rangle)$, or negative, i.e., $P(2,0 ;|\psi\rangle)<$ $P(0,2 ;|\psi\rangle)$. Finally, in Fig. 3(b) we fix the scattering phases $\left(\eta_{B}, \theta_{B}\right)$ and we calculate the asymmetry $\delta P(|\psi\rangle)$ as a function of the final state $|\psi\rangle=m\left|\psi_{A}\right\rangle+e^{i \varphi} \sqrt{1-m^{2}}\left|\psi_{B}\right\rangle$ onto which the qubit is projected. We thus obtain a nontrivial manifold of final states $|\psi\rangle$ where $\delta P(|\psi\rangle)$ is nonvanishing.

The possibility that $\delta P(|\psi\rangle) \neq 0$ is a consequence of the additional particles-qubit correlations generated by the projection of the final state $\left|\Psi_{f}\right\rangle$ onto the state $|\psi\rangle$. Finally, we notice that for classical particles [as distinct from quantum particles for which $P(2,0)=P(0,2)]$, the probabilities $P^{(c l)}(2,0)$ and $P^{(c l)}(0,2)$ associated with a beam splitter (even in the absence of a qubit) can be different. In this spirit, the effective breaking of the unitarity of the scattering matrix associated with our protocol 2 simulates the physics of classical particles in a scattering region.

\section{CONCLUSIONS}

Interaction-induced modification of quantum statistics is certainly not a new theme. Effective attractive interactions may give rise to Cooper pairs, with the corresponding scattering (depending on details of the setup) manifested by bunching [16-18]. Anyonic quasiparticles have different statistics than either fermions or bosons $[19,20,24]$. In the present work we have shown that statistical transmutation can be engineered quantum mechanically without resorting to a direct particle-particle interaction. Specifically we have considered a generalized Hong-Ou-Mandel interferometer which allows on to transmute the statistics of the scattered particles by entangling them with an external quantum degree of freedom. Our on-demand statistics transmutation is obtained through selecting the direction in Hilbert space, onto which the qubit is a posteriori projected. The entanglement between the scattered particles and the qubit may lead to the breaking of unitarity (still conserving probability). This can be manifest through a variety of interference setups. A possible experimental realization of the Hong-Ou-Mandel interferometer may involve a quantum Hall-based setup. We tune the current in any of the interferometers to be dilute; two chiral edge modes support two beams. The scattering region is realized through a quantum point contact between the two edge states. The scatterer is then coupled electrostatically to a double quantum dot (hosting an electron) representing the qubit.

\section{ACKNOWLEDGMENTS}

We acknowledge the Italia-Israel project QUANTRA, the Deutsche Forschungsgemeinschaft (Bonn) within the network CRC TR 183 (project C01) and Grant No. RO 2247/8-1, the IMOS Israel-Russia program, and the ISF. S.B. acknowledges the Institute for Theoretical Condensed Matter Physics (KIT) for support and hospitality during the completion of the work.

\section{APPENDIX A: PROTOCOL 1: EVALUATION OF THE PROBABILITY P $(n, 2-n)$}

The probabilities $P(n, 2-n)$ defined according to our protocol 1 are just the weighted average of the probabilities $P_{m}(n, 2-n)$. For this reason, in the following, we show how to calculate the probabilities

$$
P_{m}(n, 2-n)=\int d k d k^{\prime}\left|\left\langle n, 2-n ; k, k^{\prime}\left|S_{m}\right| i\right\rangle\right|^{2},
$$

where the particle initial state is $|i\rangle=a_{\mathrm{W}}^{\dagger}\left(x_{\mathrm{W}}\right) a_{\mathrm{S}}^{\dagger}\left(x_{\mathrm{S}}\right)|0\rangle$ with $a_{\ell}^{\dagger}\left(x_{\ell}\right)=\int_{0}^{\infty} d k_{\ell} \epsilon_{\ell}\left(k_{\ell}\right) e^{-i k_{\ell} x_{\ell}} a_{\ell}^{\dagger}\left(k_{\ell}\right)$ and the two-particle scattering $S_{m}=s_{m}^{(\mathrm{W})} \otimes s_{m}^{(\mathrm{S})}$ is built from the single particle scattering matrix

$$
s_{m}=\left(\begin{array}{cc}
r_{m} & t_{m}^{\prime} \\
t_{m} & r_{m}^{\prime}
\end{array}\right)=\left(\begin{array}{cc}
\sqrt{R_{m}} e^{i \theta_{m}} & \sqrt{T_{m}} e^{i \eta_{m}} \\
\sqrt{T_{m}} e^{i \eta_{m}} & -\sqrt{R_{m}} e^{i\left(2 \eta_{m}-\theta_{m}\right)}
\end{array}\right) ;
$$

we recall here that we are using the basis $\left\{a_{\mathrm{S}}^{\dagger}|0\rangle, a_{\mathrm{W}}^{\dagger}|0\rangle\right\}$ $\left(\left\{a_{\mathrm{E}}^{\dagger}|0\rangle, a_{\mathrm{N}}^{\dagger}|0\rangle\right\}\right)$ for the emitted (detected) particles. Furthermore the unitarity of $s_{m}$ implies $\left|r_{m}\right|^{2}+\left|t_{m}\right|^{2}=1,\left|t_{m}^{\prime}\right|^{2}+$ $\left|r_{m}^{\prime}\right|^{2}=1$ and $r_{m} t_{m}^{*}+t_{m}^{\prime} r_{m}^{\prime *}=0$, and we conveniently define $R_{m}=\left|r_{m}\right|^{2}=\left|r_{m}^{\prime}\right|^{2}, \quad T_{m}=\left|t_{m}\right|^{2}=\left|t_{m}^{\prime}\right|^{2} ; \quad \eta_{m}, \theta_{m} \in[0,2 \pi)$. First we evaluate the action of the two particle scattering matrix $S_{m}$ onto the particle initial state,

$$
\begin{aligned}
S_{m}|i\rangle= & \int d k_{\mathrm{W}} d k_{\mathrm{S}} \epsilon_{\mathrm{W}}\left(k_{\mathrm{W}}\right) \epsilon_{\mathrm{S}}\left(k_{\mathrm{S}}\right) e^{-i\left(k_{\mathrm{W}} x_{\mathrm{W}}+k_{\mathrm{S}} x_{\mathrm{S}}\right)} \\
& \times\left[t_{m}^{\prime} a_{\mathrm{E}}^{\dagger}\left(k_{\mathrm{W}}\right)+r_{m}^{\prime} a_{\mathrm{N}}^{\dagger}\left(k_{\mathrm{W}}\right)\right]\left[r_{m} a_{\mathrm{E}}^{\dagger}\left(k_{\mathrm{S}}\right)+t_{m} a_{\mathrm{N}}^{\dagger}\left(k_{\mathrm{S}}\right)\right]|0\rangle,
\end{aligned}
$$

then we project onto the final state $\left|1,1 ; k, k^{\prime}\right\rangle=$ $a_{N}^{\dagger}(k) a_{E}^{\dagger}\left(k^{\prime}\right)|0\rangle$ and we integrate over all possible $k$ values, obtaining

$$
\begin{aligned}
P_{m}(1,1)= & \int d k d k^{\prime}\left|\left\langle 1,1 ; k, k^{\prime}\left|S_{m}\right| i\right\rangle\right|^{2} \\
= & \int d k d k^{\prime} \mid r_{m} r_{m}^{\prime} \epsilon_{\mathrm{W}}(k) \epsilon_{\mathrm{S}}\left(k^{\prime}\right) e^{-i k x_{\mathrm{W}}} e^{-i k^{\prime} x_{\mathrm{S}}} \\
& -\left.t_{m} t_{m}^{\prime} \epsilon_{\mathrm{W}}\left(k^{\prime}\right) \epsilon_{\mathrm{S}}(k) e^{-i k^{\prime} x_{\mathrm{W}}} e^{-i k x_{\mathrm{S}}}\right|^{2} \\
= & R_{m}^{2}+T_{m}^{2}+2 R_{m} T_{m}|J|^{2},
\end{aligned}
$$

where

$$
|J|^{2}=\left|\int d k \epsilon_{\mathrm{W}}(k) \epsilon_{\mathrm{S}}^{*}(k) e^{i k\left(x_{\mathrm{W}}-x_{\mathrm{S}}\right)}\right|^{2}
$$

is the overlap integral.

The calculation of $P_{m}(2,0)$ and $P_{m}(0,2)$ can be carried out in a similar way. In this case, starting again from Eq. (A3), we can project onto $\left|2,0 ; k, k^{\prime}\right\rangle=1 / \sqrt{2} a_{N}^{\dagger}(k) a_{N}^{\dagger}\left(k^{\prime}\right)|0\rangle$, obtaining

$$
\begin{aligned}
P_{m}(2,0)= & \int d k d k^{\prime}\left|\left\langle 2,0 ; k, k^{\prime}\left|S_{m}\right| i\right\rangle\right|^{2} \\
= & R_{m} T_{m} \int d k d k^{\prime} \mid \epsilon_{\mathrm{W}}(k) \epsilon_{\mathrm{S}}\left(k^{\prime}\right) e^{-i k x_{\mathrm{W}}} e^{-i k^{\prime} x_{\mathrm{S}}} \\
& -\left.\epsilon_{\mathrm{W}}\left(k^{\prime}\right) \epsilon_{\mathrm{S}}(k) e^{-i k^{\prime} x_{\mathrm{W}}} e^{-i k x_{\mathrm{S}}}\right|^{2} \\
= & R_{m} T_{m}\left(1-|J|^{2}\right) .
\end{aligned}
$$

Similarly $P_{m}(0,2)$ can be obtained by projecting onto $\left|0,2 ; k, k^{\prime}\right\rangle=1 / \sqrt{2} a_{\mathrm{E}}^{\dagger}(k) a_{\mathrm{E}}^{\dagger}\left(k^{\prime}\right)|0\rangle ;$ the unitarity of the scattering matrix $s_{m}$ implies $P_{m}(0,2)=P_{m}(2,0)$. 


\section{APPENDIX B: PROTOCOL 2: EVALUATION OF THE PROBABILITY $P(n, 2-n ;|\psi\rangle)$}

We evaluate the probabilities

$$
\begin{gathered}
P(n, 2-n ;|\psi\rangle)=\frac{\mathcal{P}(n, 2-n ;|\psi\rangle)}{\sum_{n=0}^{2} \mathcal{P}(n, 2-n ;|\psi\rangle)}, \\
\mathcal{P}(n, 2-n ;|\psi\rangle)=\int d k d k^{\prime}\left|\sum_{m=A}^{B} \gamma_{m} a_{m}^{(n)}\left\langle\psi \mid \psi_{m}\right\rangle\right|^{2}
\end{gathered}
$$

defined according to our protocol 2. First, we evaluate the numerator of Eq. (B1) for $n=0,1,2$; explicitly we obtain

$$
\begin{aligned}
\mathcal{P}(1,1 ;|\psi\rangle)= & \left|\tilde{\gamma}_{A}\right|^{2} P_{A}(1,1)+\left|\tilde{\gamma}_{B}\right|^{2} P_{B}(1,1) \\
& +2\left|\tilde{\gamma}_{A}\right|\left|\tilde{\gamma}_{B}\right|\left[R_{A} R_{B}+T_{A} T_{B}\right. \\
& \left.+\left(R_{A} T_{B}+R_{B} T_{A}\right)|J|^{2}\right] \cos \varphi_{1,1}, \\
\mathcal{P}(2,0 ;|\psi\rangle)= & \left|\tilde{\gamma}_{A}\right|^{2} P_{A}(2,0)+\left|\tilde{\gamma}_{B}\right|^{2} P_{B}(2,0) \\
& +2\left|\tilde{\gamma}_{A}\right|\left|\tilde{\gamma}_{B}\right| \sqrt{P_{A}(2,0) P_{B}(2,0)} \cos \varphi_{2,0},
\end{aligned}
$$

$$
\begin{aligned}
\mathcal{P}(0,2 ;|\psi\rangle)= & \left|\tilde{\gamma}_{A}\right|^{2} P_{A}(0,2)+\left|\tilde{\gamma}_{B}\right|^{2} P_{B}(0,2) \\
& +2\left|\tilde{\gamma}_{A}\right|\left|\tilde{\gamma}_{B}\right| \sqrt{P_{A}(0,2) P_{B}(0,2)} \cos \varphi_{0,2},
\end{aligned}
$$

where we have introduced $\tilde{\gamma}_{m}=\gamma_{m}\left\langle\psi \mid \psi_{m}\right\rangle \equiv\left|\tilde{\gamma}_{m}\right| e^{i \arg \left(\tilde{\gamma}_{m}\right)}$, $m=A, B ; \varphi_{0}=\arg \left(\tilde{\gamma}_{A}\right)-\arg \left(\tilde{\gamma}_{B}\right)$ and

$$
\varphi_{1,1}=\varphi_{0}+2\left(\eta_{A}-\eta_{B}\right),
$$

$$
\begin{aligned}
& \varphi_{2,0}=\varphi_{0}+\eta_{A}+\theta_{A}-\left(\eta_{B}+\theta_{B}\right), \\
& \varphi_{0,2}=\varphi_{0}+3 \eta_{A}-\theta_{A}-\left(3 \eta_{B}-\theta_{B}\right) ;
\end{aligned}
$$

the probabilities $P_{m}(n, 2-n)$ with $m=A, B$ and $n=0,1,2$ are defined in Eqs. (A4) and (A6).

The denominator of Eq. (B1) turns out to be equal to

$$
\begin{array}{rl}
\sum_{n=0}^{2} & \mathcal{P}(n, 2-n ;|\psi\rangle) \\
= & \left|\tilde{\gamma}_{A}\right|^{2}+\left|\tilde{\gamma}_{B}\right|^{2}+2\left|\tilde{\gamma}_{A}\right|\left|\tilde{\gamma}_{B}\right| \\
& \times\left[\left[R_{A} R_{B}+T_{A} T_{B}+\left(R_{A} T_{B}+R_{B} T_{A}\right)|J|^{2}\right] \cos \varphi_{1,1}\right. \\
& \left.+\sqrt{P_{A}(2,0) P_{B}(2,0)}\left(\cos \varphi_{2,0}+\cos \varphi_{0,2}\right)\right] .
\end{array}
$$

In the special case where $s_{A}$ and $s_{B}$ have the same amplitudes, $P_{A}(n, 2-n)=P_{B}(n, 2-n) \equiv P(n, 2-n)$ for $n=0,1,2$. From Eq. (B5) we obtain

$$
\begin{aligned}
& \sum_{n=0}^{2} \mathcal{P}(n, 2-n ;|\psi\rangle) \\
& \quad=\left|\tilde{\gamma}_{A}\right|^{2}+\left|\tilde{\gamma}_{B}\right|^{2}+2\left|\tilde{\gamma}_{A}\right|\left|\tilde{\gamma}_{B}\right|+2 \lambda\left|\tilde{\gamma}_{A}\right|\left|\tilde{\gamma}_{B}\right| \cos \varphi_{1,1}
\end{aligned}
$$

where $\lambda \equiv P(1,1)+2 P(2,0) \cos \left(\eta_{B}-\eta_{A}+\theta_{A}-\theta_{B}\right)$; and finally, recalling Eqs. (B3a)-(B3c), we obtain $P(n, 2-$ $n ;|\psi\rangle)=\mathcal{S}(n,|\psi\rangle) P(n, 2-n)$ with

$$
\mathcal{S}(n,|\psi\rangle)=\frac{\left|\tilde{\gamma}_{A}\right|^{2}+\left|\tilde{\gamma}_{B}\right|^{2}+2\left|\tilde{\gamma}_{A}\right|\left|\tilde{\gamma}_{B}\right| \cos \varphi_{n, 2-n}}{\left|\tilde{\gamma}_{A}\right|^{2}+\left|\tilde{\gamma}_{B}\right|^{2}+2 \lambda\left|\tilde{\gamma}_{A}\right|\left|\tilde{\gamma}_{B}\right| \cos \varphi_{1,1}},
$$

which is the expression presented in the main text.
[1] W. Pauli, The connection between spin and statistics, Phys. Rev. 58, 716 (1940).

[2] W. Pauli, On the connection between spin and statistics, Progr. Theor. Phys. 5, 526 (1950).

[3] Ya. M. Blanter and M. Büttiker, Shot noise in mesoscopic conductors, Phys. Rep. 336, 1 (2000).

[4] J. Splettstösser, M. Moskalets, and M. Büttiker, Two-Particle Nonlocal Aharonov-Bohm Effect from Two Single-Particle Emitters, Phys. Rev. Lett. 103, 076804 (2009).

[5] R. Hanbury Brown and R. Q. Twiss, A new type of interferometer for use in radio astronomy, Philos. Mag. 45, 663 (1954).

[6] R. Hanbury Brown and R. Q. Twiss, A test of a new type of stellar interferometer on sirius, Nature (London) 178, 1046 (1956)

[7] P. Samuelsson, E. V. Sukhorukov, and M. Büttiker, Two-particle Aharonov-Bohm effect and Entanglement in the Electronic Hanbury Brown-Twiss Setup, Phys. Rev. Lett. 92, 026805 (2004).

[8] M. Henny, S. Oberholzer, C. Strunk, T. Heinzel, K. Ensslin, M. Holland, and C. Schönenberger, The Fermionic Hanbury Brown and Twiss experiment, Science 284, 296 (1999).

[9] S. Oberholzer, M. Henny, C. Strunk, C. Schönenberger, T. Heinzel, K. Ensslin, and M. Holland, The Hanbury Brown and Twiss experiment with fermions, Physica E 6, 314 (2000).
[10] I. Neder, N. Ofek, Y. Chung, M. Heiblum, D. Mahalu, and V. Umansky, Interference between two indistinguishable electrons from independent sources, Nature (London) 448, 333 (2007).

[11] G. Fève, A. Mahé, J.-M. Berroir, T. Kontos, B. Plaçais, D. C. Glattli, A. Cavanna, B. Etienne, and Y. Jin, An on-demand coherent single-electron source, Science 316, 1169 (2007).

[12] A. Mahé, F. D. Parmentier, E. Bocquillon, J.-M. Berroir, D. C. Glattli, T. Kontos, B. Plaçais, G. Fève, A. Cavanna, and Y. Jin, Current correlations of an on-demand single-electron emitter, Phys. Rev. B 82, 201309(R) (2010).

[13] E. Bocquillon, V. Freulon, J.-M. Berroir, P. Degiovanni, B. Plaçais, A. Cavanna, Y. Jin, and G. Fève, Coherence and indistinguishability of single electrons emitted by independent sources, Science 339, 1054 (2013).

[14] G. Burkard, D. Loss, and E. Sukhorukov, Noise of entangled electrons: Bunching and antibunching, Phys. Rev. B 61, R16303 (2000).

[15] J. Egues, G. Burkard, D. Saraga, J. Schliemann, and D. Loss, Shot noise and spin-orbit coherent control of entangled and spin-polarized electrons, Phys. Rev. B 72, 235326 (2005).

[16] J. Torrés, T. Martin, and G. B. Lesovik, Effective charges and statistical signatures in the noise of normal metalsuperconductor junctions at arbitrary bias, Phys. Rev. B 63, 134517 (2001). 
[17] J. Börlin, W. Belzig, and C. Bruder, Full Counting Statistics of a Superconducting Beam Splitter, Phys. Rev. Lett. 88, 197001 (2002).

[18] P. Samuelsson and M. Büttiker, Chaotic Dot-Superconductor Analog of the Hanbury Brown-Twiss Effect, Phys. Rev. Lett. 89, 046601 (2002).

[19] G. Campagnano, O. Zilberberg, I. V. Gornyi, D. E. Feldman, A. C. Potter, and Y. Gefen, Hanbury Brown and Twiss Interference of Anyons, Phys. Rev. Lett. 109, 106802 (2012).

[20] G. Campagnano, O. Zilberberg, I. V. Gornyi, and Y. Gefen, Hanbury Brown and Twiss correlations in quantum Hall systems, Phys. Rev. B 88, 235415 (2013).
[21] C. K. Hong, Z. Y. Ou, and L. Mandel, Measurement of Subpicosecond Time Intervals between Two Photons by Interference, Phys. Rev. Lett. 59, 2044 (1987).

[22] K. A. Ralley, I. V. Lerner, and I. V. Yurkevich, Hong-OuMandel interference with a single atom, Sci. Rep. 5, 13947 (2015).

[23] Y. V. Nazarov and Ya. M. Blanter, Quantum Transport: Introduction to Nanoscience (Cambridge University Press, Cambridge, 2009).

[24] J. M. Leinaas and J. Myrheim, On the theory of identical particles, Nuovo Cimento B 37, 1 (1977). 